der einzelnen Plaques bestrahlen und darf die oft nur scheinbar gesunde Nachbarhaut in der Nähe der Plaques nicht zu ängstlich gegen Strahlen abdecken. Insbesondere die Psoriasis des Gesichts und der Hände, die die Patienten am meisten belästigt und oft als Schönheitsfehler arbeitsunfähig macht, reagiert selir rasch auf Röntgenbestrahlungen; ein wichtiges Unterstützungsmittel der Kur sind elektrische Glühlichtbader, die alle drei Tage, wo angängig, verabreicht werden. Daß diese Glühlichtbäder den Krankheitsproze $\beta$ auch direkt beeinflussen können, konnte ich mehrfach daran feststellen, daß of t die Gefäßhaut, die im Lichtbad nicht von Strahlen getroffen wurde, ihre Krankheitsherde nuch behalten hatte, wo unter Strahlenwirkung die anderen Plaques längst abgeheilt waren. Indessen habe ich nicht feststellen können, daß Bogenlicht-oder Sonnenbäder besser wirkten als gewöhnliche Glühlichtbäder, sodaß auch hier die Bewertung der ultravioletten Strahlen als Eleilfaktor noch weiter erforscht werden mub.

\title{
Korrespondenzen.
}

\section{Zur Behandlung der Schuppenflechte mit Ultraviolett-} strahlen.

\section{Bemerkung zum Artikel von Dr. Becker in No. 51 dieser} Wochenschrift (1907).

Von Dr. Felix Davidsobn, Arzt für Lichtbehandlung, in Berlin.

Becker betont die günstige Beeinflussung der Psoriasis durch ultraviolette Strahlen, und empfiehlt sie neben der Röntgenbestrahlung als gleichwertig. An einem Material von uber 100 Fällen (in den letzten zwei Jahren) bin ich jedoch zu einer anderen Bewertung dieser Therapie gekommen. Die Behandlung mit der Uviollampe habe ich, da sie eine Sitzungsdauer bis 30 Minuten and noch langer erfordert als zu zeitraubend verlassen, auch versagte sie bei dickschwartigen Fällen häufig gänzlich. Die Quarzlampenbehandlung kommt ja nur für kleinere Herde in Betracht und leistet bei k urzerer Bestrahlungsdauer oft recht Gutes; jedoch kann auch diese Methode sich nicht mit der Röntgentherapie messen, die in kürzester Zeit die besten Resultate erzielt; allerdings muß man täglich etwa fünf Minuten mit mittelweicher bis harter Röhre unter Wechsel 